\title{
Antimonial drugs entrapped into phosphatidylserine liposomes: physicochemical evaluation and antileishmanial activity
}

\author{
Samanta Etel Treiger Borborema ${ }^{[1],[2], ~ J o a ̃ o ~ A l b e r t o ~ O s s o ~ J u n i o r ~}{ }^{[3]}$, \\ Heitor Franco de Andrade Junior ${ }^{[4]}$ and Nanci do Nascimento ${ }^{[1]}$
}

[1]. Centro de Biotecnologia, Instituto de Pesquisas Energéticas e Nucleares, São Paulo, São Paulo, Brasil. [2]. Centro de Parasitologia e Micologia, Instituto Adolfo Lutz, São Paulo, São Paulo, Brasil. [3]. Centro de Radiofarmácia, Instituto de Pesquisas Energéticas e Nucleares, São Paulo, São Paulo, Brasil. [4]. Laboratório de Protozoologia, Instituto de Medicina Tropical de São Paulo, Universidade de São Paulo, São Paulo, São Paulo, Brasil.

\begin{abstract}
Introduction: Leishmaniasis is a disease caused by the protozoan Leishmania that resides mainly in mononuclear phagocytic system tissues. Pentavalent antimonials are the main treatment option, although these drugs have toxic side effects and high resistance rates. A potentially alternative and more effective therapeutic strategy is to use liposomes as carriers of the antileishmanial agents. The aims of this study were to develop antimonial drugs entrapped into phosphatidylserine liposomes and to analyze their biological and physicochemical characteristics. Methods: Liposomes containing meglumine antimoniate (MA) or pentavalent antimony salt (Sb) were obtained through filter extrusion (FEL) and characterized by transmission electron microscopy. Promastigotes of Leishmania infantum were incubated with the drugs and the viability was determined with a tetrazolium dye (MTT assay). The effects of these drugs against intracellular amastigotes were also evaluated by optical microscopy, and mammalian cytotoxicity was determined by an MTT assay. Results: Liposomes had an average diameter of $162 \mathrm{~nm}$. MA-FEL showed inhibitory activity against intracellular $L$. infantum amastigotes, with a $50 \%$ inhibitory concentration $\left(\mathrm{IC}_{50}\right)$ of $0.9 \mu \mathrm{g} / \mathrm{mL}$, whereas that of MA was $60 \mu \mathrm{g} / \mathrm{mL}$. Sb-FEL showed an $\mathrm{IC}_{50}$ value of $0.2 \mu \mathrm{g} / \mathrm{mL}$, whereas that of free Sb was $9 \mu \mathrm{g} / \mathrm{mL}$. MA-FEL and Sb-FEL had strong in vitro activity that was 63 -fold and 39-fold more effective than their respective free drugs. MA-FEL tested at a ten-times higher concentration than Sb-FEL did not show cytotoxicity to mammalian cells, resulting in a higher selectivity index. Conclusions: Antimonial drug-containing liposomes are more effective against Leishmania-infected macrophages than the non-liposomal drugs.
\end{abstract}

Keywords: Antimony. Leishmania infantum. Liposome. Meglumine antimoniate. Phosphatidylserine.

\section{INTRODUCTION}

The protozoan parasite Leishmania is responsible for a spectrum of diseases ranging from self-limiting cutaneous lesions to disseminating diffuse cutaneous, mucocutaneous, and visceral infections that can be fatal if left untreated. Leishmaniasis is one of the most important neglected tropical diseases that affect 350 million people in 98 countries, with a global incidence of 0.9-1.6 million cases per year, and visceral leishmaniasis leads to $20,000-40,000$ deaths annually ${ }^{(1)}$. The disease burden is calculated at 2,356,000 disability-adjusted life years, holding a significant rank among communicable diseases ${ }^{(2)}$.

Leishmaniasis control relies on integrated vector management, and early and accurate diagnosis and treatment of human cases. The treatment is based on specific antileishmanial

Corresponding author: Dra. Samanta Etel Treiger Borborema.

e-mail: samantaborborema@gmail.com

Received 5 February 2016

Accepted 13 April 2016 drugs. Pentavalent antimonials such as sodium stibogluconate (Pentostam ${ }^{\circledR}$ ) and meglumine antimoniate (MA, Glucantime ${ }^{\circledR}$ ) have been used as the first-line treatment against all forms of leishmaniasis for more than 70 years. However, they have several limitations owing to toxicity, treatment failures, long treatment duration, and drug resistance ${ }^{(3)}$. Thus, there is a continued need for new leishmaniasis therapies that are safe, effective in inducing a long-term cure, and easy to administer.

Drug discovery and development in the area of parasitic diseases progress at a very slow rate owing to the general lack of economic investment. Considering this limitation, an approach based on the improvement of existing drugs has been more successful than those based on designing new chemical entities ${ }^{(4)}$. Given that Leishmania parasites colonize macrophages, which are responsible for the clearance of liposomes, the use of liposomes has been studied for many years as an efficient strategy for the delivery of antileishmanial agents to Leishmania-infected tissues and reducing the parasite $\operatorname{load}^{(5)(6)}$. Liposome-encapsulated antimonials were found to be hundreds-fold more effective than the corresponding free drugs for treating experimental visceral leishmaniasis ${ }^{(7)}$, and could promote suppression of the parasite in the liver and/or spleen ${ }^{(8)}$. 
Interestingly, liposome-encapsulated antimonials were also effective against cutaneous leishmaniasis, in which the parasites are located in peripheral tissues rather than in the liver ${ }^{(9)}$.

Liposomes represent the most appropriate drug delivery system for antimonials, because of their natural tendency to be taken up through the mononuclear phagocytic system, their relative safety, high versatility with respect to lipid composition, the volume and composition of the internal compartment, and the vesicle size and lamellarity ${ }^{(10)}$. An effective strategy for controlling the stability and reactivity of a liposome can be achieved by incorporation of negatively charged phospholipids. Charged liposomes are known to be phagocytosed at higher rates owing to specific or electrostatic interactions between cells and vesicles ${ }^{(11)}$. We previously reported that inclusion of phosphatidylserine (PS) can lead to the preferred recognition of liposomes by macrophages and improve drug encapsulation efficiency ${ }^{(12)}$. Although previous studies have shown the antileishmanial activity of MA-containing liposomes ${ }^{(12)(13)}$, other aspects related to their physicochemical characteristics and encapsulation of antimony salt are unknown.

Thus, as part of our continuous investigation of the properties of MA-containing liposomes, the aims of this study were to develop antimonials entrapped in PS liposomes, and analyze their physicochemical characteristics and antileishmanial activity in Leishmania (Leishmania) infantum chagasi-infected macrophages in vitro.

\section{METHODS}

\section{Chemicals}

Egg-hydrogenated phosphatidylcholine (PC) and PS were kindly provided by Lipoid GmbH. Glycerol, sodium dodecyl sulfate, methanol, chloroform, and antimony Inductively Coupled Plasma (ICP) standard traceable to Standard Reference Materials (SRM) were purchased from Merck. Dimethyl sulfoxide, cholesterol, 3-(4,5-dimethylthiazol-2-yl]2,5-diphenyltetrazolium bromide dye (thiazolyl blue; MTT), potassium hexahydroxoantimonate (V) (pentavalent antimony salt; Sb), M-199 medium, Roswell Park Memorial Institute (RPMI) 1640 medium (without phenol red), dialysis tubing, and the cellulose membrane were purchased from Sigma. Fetal bovine serum (FBS) was obtained from Gibco and phosphotungstic acid from Vetec Quimica. Polycarbonate membranes were purchased from Avanti Lipids. MA (Glucantime ${ }^{\circledR} ; 300 \mathrm{mg} / \mathrm{mL}$ ) was obtained from Sanofi-Aventis.

\section{Ethical considerations}

Experimental animals: golden hamsters (Mesocricetus auratus) and BALB/c mice were supplied by the animal breeding facility at the Faculty of Medicine, University of São Paulo, and were maintained in sterilized cages in a controlled environment with free access to water and food. All animal procedures were performed with the approval of the Research Ethical Committee [Comitê de Ética em Pesquisa (CEP)] of the Tropical Medicine Institute of São Paulo [Instituto de Medicina Tropical de São Paulo (IMTSP)]: CEP-IMTSP 012/29/042008.

\section{Parasites and macrophages}

Leishmania infantum (MHOM/BR/1972/LD) promastigotes were maintained in M-199 medium supplemented with $10 \%$ FBS and $0.25 \%$ hemin at $24^{\circ} \mathrm{C}$. Leishmania infantum was maintained in the golden hamsters for up to 60-70 days after infection. Amastigotes were obtained from spleens of previously infected hamsters by maceration of the tissue and purification by differential centrifugation.

Peritoneal macrophages were collected from the peritoneal cavities of BALB/c mice by washing with $10 \%$ FBS-supplemented RPMI 1640. The cells were maintained at $37^{\circ} \mathrm{C}$ in $5 \% \mathrm{CO}_{2}{ }^{(14)}$.

\section{Preparation and characterization of liposomes-encapsulated antimonials}

Liposomes were prepared by filter extrusion through polycarbonate membranes (FEL) ${ }^{(13)}$. They were composed of PC, cholesterol, and PS (molar ratio of 5:4:1), at a final lipid concentration of $111 \mathrm{mg} / \mathrm{mL}$. The lipids were mixed in a chloroform:methanol $(2: 1 \mathrm{v} / \mathrm{v})$ solution, and dried to a lipid film with a rotary evaporator at $55^{\circ} \mathrm{C}$ under controlled vacuum. The dry lipid film was then dispersed in $1 \mathrm{~mL}(81 \mathrm{mg} / \mathrm{mL})$ of MA or $2 \mathrm{~mL}(25 \mathrm{mg} / \mathrm{mL})$ of $\mathrm{Sb}$ at $55^{\circ} \mathrm{C}$ for $50 \mathrm{~min}$. After vesicle hydration, the solution was sonicated in a sonicating bath at $55^{\circ} \mathrm{C}$ for $10 \mathrm{~min}$ and then subjected to repetitive extrusion $(17 \times)$ through polycarbonate membranes with a $200-\mathrm{nm}$ pore size, by using a mini-extruder device Liposofast (Avestin Inc.). Drugcontaining liposomes were separated from the non-encapsulated drug by 24 -h dialysis (14kDa molecular weight cut-off) at $4^{\circ} \mathrm{C}$ in isotonic glycerol solution (IGS). Empty liposomes with an identical phospholipid composition were prepared using the same method as those containing drugs, and were used as controls.

The amount of antimony in the resulting liposome suspension was determined by instrumental neutron activation analysis (INAA) ${ }^{(12)}$. Samples were irradiated at the IEA-R1 Research Reactor at Comissão Nacional de Energia NuclearInstituto de Pesquisas Energéticas e Nucleares, São Paulo (CNEN-IPEN/SP) together with solutions of the Sb standard. Encapsulation efficiency was determined by measuring antimony concentrations in the liposomal dispersions before and after separation of the unencapsulated drug. The values were calculated as the percentage of the drug entrapped into the liposomes. The final phospholipid concentration was determined using the Stewart assay ${ }^{(15)}$.

The average diameter of the liposomes was determined by negative-staining transmission electron microscopy using $1 \%$ phosphotungstic acid. Samples were examined under a JEM-1010 transmission electron microscope (JEOL).

Antimony release from liposomes was evaluated in IGS and normal hamster pooled plasma. An appropriate amount of liposomes was suspended in IGS (pH 7.4) and incubated at $4^{\circ} \mathrm{C}$. Likewise, to mimic the physiological conditions, normal hamster pooled plasma was supplemented with liposomes and incubated at $37^{\circ} \mathrm{C}$. After incubation periods of 15,60 , and $120 \mathrm{~min}$, the samples were removed and centrifuged 
$\left(14,000 \times g, 30 \mathrm{~min}\right.$, at $\left.4^{\circ} \mathrm{C}\right)$ to remove the leaked drugs. Drug concentrations in the pellet and supernatant were determined by INAA. Drug release was expressed as the percentage of the concentration of the encapsulated MA.

\section{Determination of activity against Leishmania infantum}

To determine the $50 \%$ inhibitory concentration $\left(\mathrm{IC}_{50}\right)$ against L. infantum, promastigotes were seeded in 96-well microplates at a density of $1 \times 10^{6} \mathrm{cells} / \mathrm{well}$. Drugs were diluted with growth medium and incubated with the parasites at $24^{\circ} \mathrm{C}$ for $24 \mathrm{~h}$. Parasite viability was determined using the colorimetric MTT assay ${ }^{(16)}$. The assay involves the conversion of the water-soluble MTT to an insoluble formazan. Formazan is then solubilized, and the concentration is determined by measuring the optical density at $570 \mathrm{~nm}$. For the analysis, $100 \%$ viability was defined based on the optical density of the promastigotes incubated without drugs (control) after normalization ${ }^{(14)}$.

Activity against intracellular L. infantum amastigotes was determined in infected macrophages. Macrophages were isolated from the peritoneal cavities of BALB/c mice, seeded into 24-well plates containing glass cover slips at $4 \times 10^{5}$ cells/ well, and incubated at $37^{\circ} \mathrm{C}$ for $24 \mathrm{~h}$. Leishmania infantum amastigotes were isolated from the spleens of previously infected hamsters, purified by differential centrifugation, and added to the macrophages at a ratio of 10:1 (amastigotes: macrophages). The plates were further incubated for $24 \mathrm{~h}$. Non-internalized parasites were removed by washing once with medium and the cells were then incubated with the drugs for five days at $37^{\circ} \mathrm{C}$ in $5 \% \mathrm{CO}_{2}$. The cells were fixed in methanol, stained with Giemsa stain, and observed under a light microscope. The number of infected macrophages was determined among 400 macrophages observed in the drug-treated and untreated cells. The number of infected macrophages in the untreated cultures was considered $100 \%$ for calculating the percentage of infection in the drugtreated cultures ${ }^{(12)}$.

\section{Determination of cytotoxicity against macrophages}

Peritoneal macrophages were seeded at $4 \times 10^{5} /$ well in 96-well microplates and incubated with the drugs for $48 \mathrm{~h}$ at $37^{\circ} \mathrm{C}$ in $5 \% \mathrm{CO}_{2}{ }^{(14)}$. The $50 \%$ cytotoxic concentration $\left(\mathrm{CC}_{50}\right)$ of the test compounds was determined using a colorimetric MTT assay. The selectivity index (SI) was calculated using the following equation: $\mathrm{SI}=\mathrm{CC}_{50}$ (Macrophages) $/ \mathrm{IC}_{50}$ (Leishmania amastigotes).

\section{Statistical analysis}

The data obtained are reported as the mean and standard deviation of duplicate samples from two or three independent assays. $\mathrm{IC}_{50}$ and $\mathrm{CC}_{50}$ values were calculated using sigmoid dose-response curves generated using GraphPad Prism 5.03 and the $95 \%$ confidence intervals.

\section{RESULTS}

\section{Liposome characterization}

Liposome formulations containing two different antimonials, MA and $\mathrm{Sb}$, were obtained through filter extrusion. The physicochemical characteristics of the liposomes were evaluated following drug entrapment (Table 1). The lipid content was determined by the colorimetric Stewart assay: the MA-FEL and Sb-FEL formulations had a mean phospholipid content of 35mg/ $\mathrm{mL}$ and $21 \mathrm{mg} / \mathrm{mL}$, respectively. As determined by INAA, the average mass of $\mathrm{Sb}$ for the MA-FEL and Sb-FEL liposomes was $9.5 \mathrm{mg}$ and $1.3 \mathrm{mg}$, respectively. The antimony/lipid ratio was 0.74 and $0.45(\mathrm{w} / \mathrm{w})$ for MA-FEL and Sb-FEL, respectively. The encapsulation efficiency, representing the amount of drug entrapped into FEL relative to the initial concentration of the drug used in the preparation of liposomes, showed a mean value of $11.7 \%$ and $2.9 \%$ for MA-FEL and Sb-FEL, respectively.

The morphological characterization of liposomes on the day of preparation was analyzed by transmission electron microscopy. Spherical-shaped vesicles were predominant and the liposomes were multilamellar (Figure 1A). Size measurement of the liposomes indicated an average diameter of $162 \mathrm{~nm}$ (Figure 1B). Size reduction and population homogeneity were achieved by sequential repeated extrusion through the polycarbonate membranes.

Considering that liposomes should be used as soon as prepared, drug release was assessed in two different conditions: in storage condition in a buffer solution at $4{ }^{\circ} \mathrm{C}$, and in physiological condition in normal hamster pooled plasma at $37^{\circ} \mathrm{C}$. As shown in Figure 2, liposomes were slightly more stable in the IGS storage condition, and approximately $99 \%$ of the entrapped drug was retained at the end of $2 \mathrm{~h}$. The MAFEL incubated in physiological condition lost about $10 \%$ of its content in $1 \mathrm{~h}$, after which the drug release was stabilized; the MA-FEL retained approximately $90 \%$ of the entrapped drug at the end of $2 h$.

TABLE 1 - Characteristics of liposome-encapsulated antimonial drugs.

\begin{tabular}{lcccc}
\hline Liposome & $\begin{array}{c}\text { Sb/lipid } \\
(\mathbf{w} / \mathbf{w})\end{array}$ & $\begin{array}{c}\text { Encapsulated Sb } \\
(\mathbf{m g})\end{array}$ & $\begin{array}{c}\text { Encapsulation } \\
\text { efficiency (\%) }\end{array}$ & $\begin{array}{c}\text { Mean diameter } \\
(\mathbf{n m})\end{array}$ \\
\hline MA-FEL & 0.74 & $9.50 \pm 1.44$ & $11.73 \pm 1.77$ \\
Sb-FEL & 0.45 & $1.34 \pm 0.19$ & $2.96 \pm 0.06$ \\
\hline
\end{tabular}

Sb: pentavalent antimony; MA-FEL: meglumine antimoniate-containing liposomes; Sb-FEL: pentavalent antimony-containing liposomes; w/w:weight/weight; nd: not determined; SD: standard deviation. The results are the mean \pm SD from three independent experiments. 


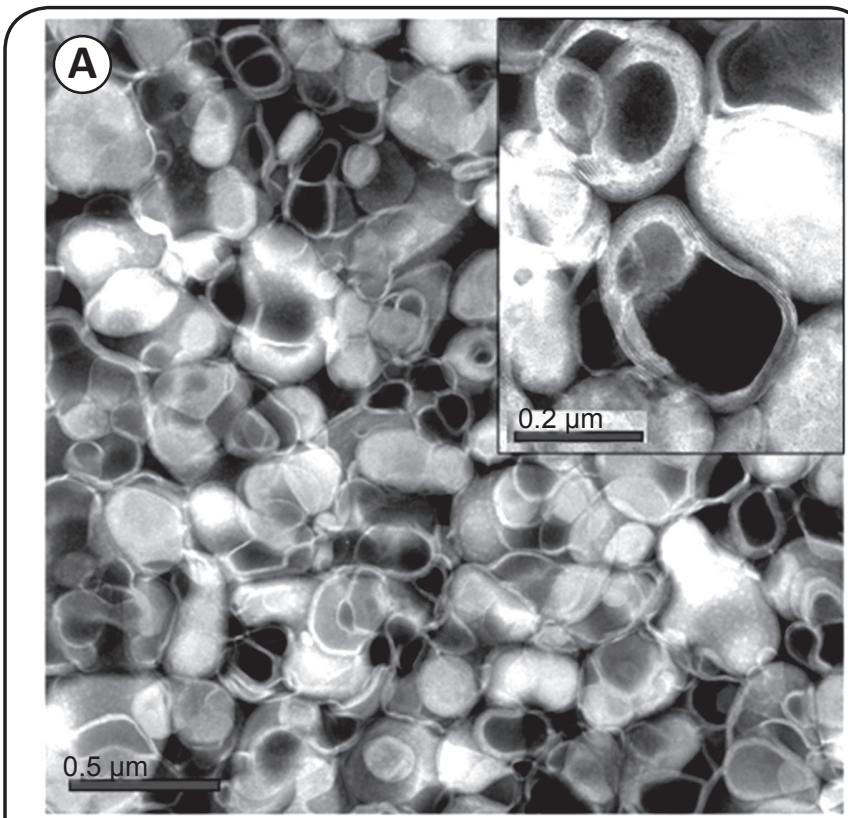

(B)

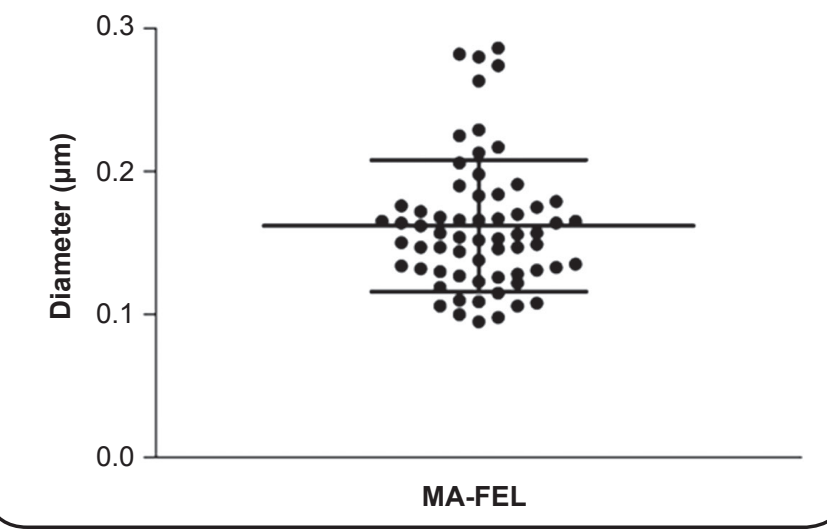

FIGURE 1 - Negative-staining transmission electron microscopy analysis of meglumine antimoniate-containing liposomes. A. Representative TEM image of liposomes. B. Graph showing the particle size distribution. MA-FEL: meglumine antimoniate-containing liposomes; TEM: transmission electron microscopy.

\section{Antileishmanial and cytotoxic activity of liposomes-encapsulated antimonials}

Meglumine antimoniate and $\mathrm{Sb}$ were entrapped in liposomes and evaluated for their relative antileishmanial activity. The drugs were tested for their ability to kill $L$. infantum promastigotes by using an MTT assay. As summarized in Table 2, evaluation of the $\mathrm{IC}_{50}$ values demonstrated that the promastigotes were not susceptible to free MA or the liposome-encapsulated drugs. Instead, promastigotes were susceptible to the $\mathrm{Sb}$ salt solution $\left(\mathrm{IC}_{50}=67.61 \mu \mathrm{g} / \mathrm{mL}\right)$.

The activity of the free and liposome-encapsulated drugs in intracellular Leishmania amastigote-infected macrophages was observed after $120 \mathrm{~h}$ by light microscopy. The doseresponse curves for the determination of the $\mathrm{IC}_{50}$ values of

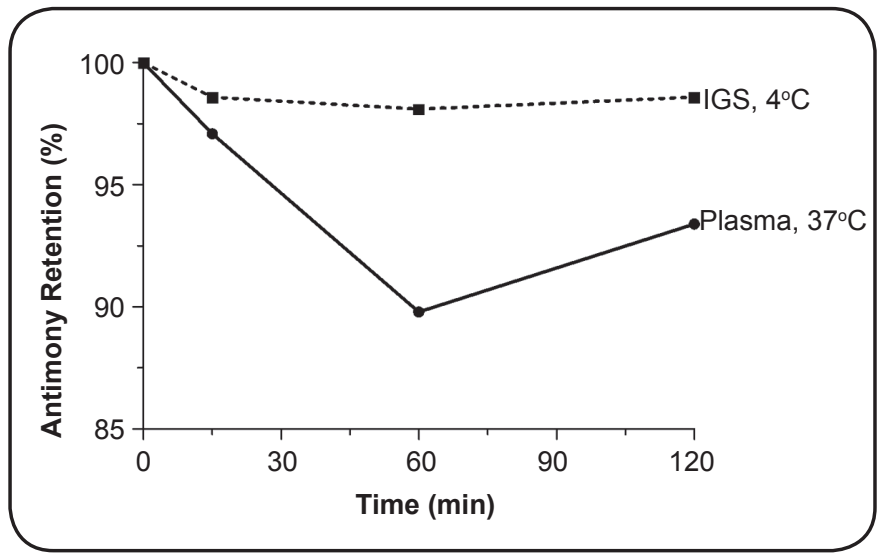

FIGURE 2 - In vitro release of MA-FEL. Liposomes were incubated in IGS at $4^{\circ} \mathrm{C}(\square)$ and in plasma at $37^{\circ} \mathrm{C}(\bullet)$. At the times indicated, the samples were centrifuged and the antimony concentration was determined by INAA. Values represent means from a single experiment. IGS: isotonic glycerol solution; MA-FEL: meglumine antimoniate-containing liposomes; INAA: instrumental neutron activation analysis.

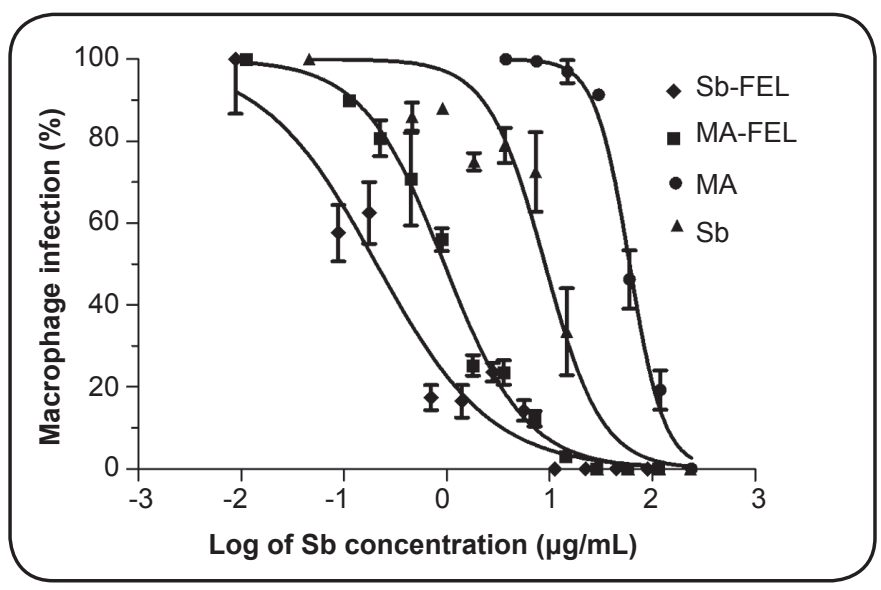

FIGURE 3 - Dose-response curve for determination of the $\mathrm{IC}_{50}$ of $\mathrm{MA}$ and MA-FEL, salt solution $\mathrm{Sb}$, and Sb-FEL in mouse peritoneal macrophages infected with Leishmania (Leishmania) infantum chagasi amastigotes. Cells were treated for a total of 5 days. Data points represent the mean \pm SD of duplicate samples from a single experiment, representative of three different experiments. Sb-FEL: pentavalent antimony-containing liposomes; MA-FEL: meglumine antimoniate-containing liposomes; MA: meglumine antimoniate; Sb: pentavalent antimony; $\mathbf{I C}_{50}: 50 \%$ inhibitory concentration; SD: standard deviation.

drugs in amastigotes are shown in Figure 3. Analysis of the antileishmanial activity revealed that MA-FEL treatment inhibited the parasite with an $\mathrm{IC}_{50}$ value of $0.95 \mu \mathrm{g} / \mathrm{mL}$, whereas the $\mathrm{IC}_{50}$ value for free MA was $60.28 \mu \mathrm{g} / \mathrm{mL}$ (Table 2). Furthermore, free and encapsulated $\mathrm{Sb}$ solutions were more active in the amastigotes than MA. Sb-FEL showed an $\mathrm{IC}_{50}$ value of $0.21 \mu \mathrm{g} / \mathrm{mL}$, whereas the $\mathrm{IC}_{50}$ value for free $\mathrm{Sb}$ was $9.05 \mu \mathrm{g} / \mathrm{mL}$.

Macrophages were incubated with the drugs for $48 \mathrm{~h}$ to evaluate the in vitro cytotoxicity using the MTT assay. Liposome-encapsulated drugs and free MA did not show cytotoxicity. However, free $\mathrm{Sb}$ was very toxic, with a $\mathrm{CC}_{50}$ value 
TABLE 2 - In vitro antileishmanial activity and cytotoxicity of MA, MA-FEL, salt solution Sb, and Sb-FEL.

\begin{tabular}{|c|c|c|c|c|}
\hline Drug & \multicolumn{2}{|c|}{$\mathrm{IC}_{50}(95 \% \mathrm{CI}) *(\mu \mathrm{g} / \mathrm{mL})$} & \multicolumn{2}{|c|}{$\mathrm{CC}_{50}(95 \% \mathrm{CI}) *(\mu \mathrm{g} / \mathrm{mL})$} \\
\hline MA-FEL & na & $\begin{array}{c}0.95 \\
(0.86-1.04)\end{array}$ & $>1,000$ & $>1,053$ \\
\hline $\mathrm{Sb}-\mathrm{FEL}$ & na & $\begin{array}{c}0.21 \\
(0.16-0.27)\end{array}$ & $>100$ & $>435$ \\
\hline MA & na & $\begin{array}{c}60.28 \\
(56.22-64.64)\end{array}$ & $>1,000$ & $>17$ \\
\hline $\mathrm{Sb}$ & $\begin{array}{c}67.61 \\
(56.41-81.02)\end{array}$ & $\begin{array}{c}9.05 \\
(7.48-10.94)\end{array}$ & $\begin{array}{c}19.84 \\
(18.16-21.67)\end{array}$ & 2.2 \\
\hline
\end{tabular}

MA: meglumine antimoniate; MA-FEL: meglumine antimoniate-containing liposomes; Sb: pentavalent antimony; Sb-FEL: pentavalent antimony-containing liposomes; $\mathbf{I C}_{\mathbf{5 0}}$ (95\% CI): $50 \%$ inhibitory concentration (95\% confidence interval); $\mathbf{C C}_{\mathbf{5 0}}$ (95\% CI): 50\% cytotoxic concentration ( $95 \%$ confidence interval); L.: Leishmania SI: selectivity index $\left(\mathrm{CC}_{50}\right.$ peritoneal macrophage/ $\mathrm{IC}_{50}$ amastigotes); na: not active. *Concentration is based on the total antimony amount. The values represent a single experiment, representative of three performed.

of $19.84 \mu \mathrm{g} / \mathrm{mL}$ and a selectivity index of 2.2. MA-FEL presented higher selectivity than Sb-FEL, with a selectivity index of $>1,053$ and $>435$, respectively. In addition, morphological observations showed that empty liposomes did not have antileishmanial activity and toxicity toward macrophages when incubated with an equivalent amount of liposome, compared with liposome-encapsulated drugs.

\section{DIscussion}

This study demonstrated that antimonial-containing PS liposomes were able to eliminate intracellular L. infantum amastigotes. MA-FEL showed strong activity against intracellular amastigotes and was 63 -fold more effective than the free drug. As expected, L. infantum promastigotes were not susceptible to the free drug or MA-FEL. By contrast, $\mathrm{Sb}$ was active against $L$. infantum promastigotes, but not in the liposome formulation. Furthermore, Sb-FEL showed strong activity against intracellular amastigotes and was 39-fold more effective than the free drug. L. infantum amastigotes were 4-fold more susceptible to Sb-FEL than to MA-FEL. However, MA-FEL tested at a 10-fold higher concentration than that of Sb-FEL did not show cytotoxicity to mammalian cells, resulting in a selectivity index higher than 1053. Sb is known to be active against both life stages and is more potent than sodium stibogluconate $^{(17)}$.

Our $\mathrm{IC}_{50}$ values are consistent with the findings described by Tempone et al. ${ }^{(13)}$, who showed that the MA-PS-liposome was 16-fold more effective than the free drug in reducing the parasite burden in macrophages. We have also observed similar results using liposomes with or without PS, which were $\geq 10$ fold more effective than the free drug against L. major-infected macrophages ${ }^{(12)}$. Our findings emphasize that liposomal MA is more effective than the free drug as well as $\mathrm{Sb}$ in either form against Leishmania-infected macrophages.
Liposomes have been used to improve the therapeutic approaches for leishmaniasis. Drug-free PC-stearylamine (SA) liposomes have been reported to be active against $L$. donovani promastigotes in an in vivo model ${ }^{(18)}$, based on the hypothesis that PC-SA cationic liposomes damage Leishmania promastigotes and amastigotes primarily via their interaction with surface PS, leading to membrane disruption ${ }^{(19)}$; however, this formulation may be toxic. Miltefosine entrapped in the same liposomal formulation improved the susceptibility of miltefosine-resistant Leishmania promastigotes, whereas this formulation was completely inactive against amastigotes ${ }^{(20)}$. Liposomes containing paromomycin were found to be three to four times more effective against L. major promastigotes and amastigotes than paromomycin alone, and infected mice were completely cured after topical treatment ${ }^{(21)}$.

The lipid composition and charge surface of the liposomes may influence the efficacy of treatment. Positively charged PC liposomes containing MA were found to be less effective than negatively charged liposomes. In contrast, positively and negatively charged sphingomyelin liposomes were equally effective. Liposomes containing phosphatidylserine were among the less effective preparations, due to the fact that they had a much higher surface charge density ${ }^{(22)}$. To our knowledge, PS liposomes containing MA were preferentially taken up by infected rather than by uninfected macrophages, probably due to changes in phagocytic behavior after infection ${ }^{(12)}$. Another interesting observation was that the liposomes were localized close to the amastigotes when observed microscopically ${ }^{(12)}$. PS liposomes were taken up to a 10 -fold higher extent than neutrally charged liposomes by a perfused liver ${ }^{(23)}$.

Phosphatidylserine liposomes are efficiently eliminated from the blood by cells of the mononuclear phagocytic system, predominantly Kupffer cells in the liver. Hepatocytes as well as liver endothelial cells participate in the elimination process, with scavenger receptors being involved in the uptake ${ }^{(23)}$. 
Followed by endocytic internalization, inside the macrophages, these anionic liposomes probably exert dual effects by interacting directly with the intracellular parasites and also inducing macrophage microbicidal activity ${ }^{(24)}$. It is hypothesized that after internalization by phagocytosis, liposomes are degraded by lysosomal phospholipases, and the antimony is released within the phagolysosome of the macrophage, where Leishmania parasites live and multiply; thus, the parasites can be excreted or diffuse through the cytosol ${ }^{(25)}$. In the latter case, it is hypothesized that antimony promotes amastigote death by interfering with diverse cellular processes such as the formation of stable thiol complexes, inhibition of trypanothione reductase, and binding to zinc finger proteins, leading to irreversible cell damage ${ }^{(10)}$.

Liposome characterization is important since it provides information about differences in structure caused by changes in the method of preparation and lipid composition. These aspects in turn affect vesicle behavior, both in vitro (stability) and in vivo (disposition). Therefore, a proper identification of the structure is essential to obtain reproducible results, which is a prerequisite for the successful introduction of liposomes in therapy ${ }^{(26)}$. Liposome type, size, lipid composition, membrane fluidity, stability, charge, and ease of preparation are factors that must be considered when designing liposomal carriers.

The hydration of a lipid film with an antimonial followed by sonication and filter extrusion through polycarbonate membranes was the method of choice to prepare the liposome formulations. Transmission electron microscopy analysis showed the homogeneity and size of MA encapsulated in the liposome. Size reduction and reduced polydispersity were achieved by extrusion through polycarbonate membrane filters as proposed by Olson et al. ${ }^{(27)}$ MA-FEL exhibited vesicles with a homogeneous size distribution with a mean diameter of $162 \mathrm{~nm}$, which is close to the pore size of the polycarbonate membrane that was used to extrude it $(200 \mathrm{~nm})$. This process is an advantageous approach with respect to the production of multilamellar vesicles with a defined and well-characterized size distribution. Extrusion is easy, reproducible, does not introduce impurities into the vesicles, and causes no detectable lipid degradation ${ }^{(27)}$.

The vesicle size influences the clearance rate and biodistribution of a liposome; it has been shown that large multilamellar vesicles enhance delivery to the lung, whereas small unilamellar vesicles appear to exhibit increased partitioning to the bone marrow. Moreover, decreasing the vesicle size increases the longevity of liposomes in the circulation ${ }^{(28)}$.

The extents of drug entrapment and retention as well as their influencing factors are important considerations in the design of liposome-mediated drug delivery systems. Antimonials are hydrophilic drugs that are entrapped in the aqueous compartment. MA-FEL showed an encapsulation efficiency of approximately $12 \%$, whereas Sb-FEL had a smaller value of $3 \%$. The encapsulation efficiency for Sb-entrapped liposomes varied from 8 to $50 \%$, depending on the preparation method ${ }^{(4)}$. Trivalent antimony entrapped in a liposome prepared by filter extrusion showed an average encapsulation efficiency of $15 \%{ }^{(29)}$. Incorporation of charged lipids into bilayers has been shown to increase the aqueous volume in liposomes ${ }^{(30)}$, which was probably due to charge repulsion separating the adjacent bilayers. We previously showed that MA encapsulated in neutral liposomes had an encapsulation efficiency of $25 \%$, whereas the efficiency increased to $38 \%$ when using PS-containing liposomes ${ }^{(12)}$.

Another important consideration for the use of liposomes as pharmaceuticals concerns the stability of the sample from the time of drug encapsulation until their use in vivo. MA-FEL's stability was higher when incubated in an isotonic solution than in plasma. The interaction between the liposomes and plasma components destabilizes liposomes and results in more drug leakage ${ }^{(31)}$. Liposomes usually interact in vivo with distinct plasma proteins such as albumin, complement and related proteins, immunoglobulins, fribonectin, C-reactive protein, apoliproteins, glycoprotein, lipoproteins, and the so-called opsonins, which bind to the surface of vesicles and mediate their endocytosis by macrophages ${ }^{(32)}$. However, this formulation was found to be stable when incubated in buffer under storage condition.

To date, liposomal amphotericin B (AmBisome $\left.{ }^{\circledR}\right)$ is the only liposomal product approved for the treatment of visceral leishmaniasis in adult and pediatric patients. AmBisome is considered as the first choice for treating patients who are unresponsive to antimonials ${ }^{(33)}$. A recent agreement between the World Health Organization and the manufacturer resulted in a reduction of the price of AmBisome for endemic regions. Even with preferential pricing, liposomal amphotericin B is not as costeffective as other first-line regimens, and thus not affordable for all patients, who are primarily inhabitants of developing countries ${ }^{(34)}$.

The main expected benefits for the use of antimonial liposome-based therapy would be the reduction of the amount of antimony and related side effects, enhanced drug effectiveness, and improved patient compliance. It is hypothesized that liposomal therapy, when compared with conventional therapy, may reduce the risk of drug resistance by promoting a very high drug concentration at the target site starting from the first dose and a shorter treatment course ${ }^{(4)}$. Furthermore, the short- versus long-course therapy would considerably reduce the costs related to hospitalization and laboratory monitoring. Therefore, the use of liposomes seems to be the most effective and advanced approach for improving antimonial chemotherapy, allowing for a reduction in the dose and frequency of dosing. As previously estimated, a single dose of antimonial liposome therapy would cost less than the estimated cost of a single AmBisome infusion ${ }^{(4)}$.

In conclusion, considering Sb-FEL's lower encapsulation efficiency and higher cytotoxicity, MA-FEL appears to be a better choice. These data demonstrate the increased effectiveness of liposome-encapsulated MA against intracellular L. infantum amastigotes compared with the free standard therapeutic drug, resulting in an improvement of the selectivity index. Nevertheless, further in vivo studies are required to evaluate both the specific interactions of the liposomes with the parasites and host cells and the therapeutic activity of liposomal MA. Furthermore, this work highlights the general potential of liposome-encapsulated antileishmanial agents for drug targeting to increase efficacy and reduce the dose required for effective therapy. 


\section{CONFLICT OF INTEREST}

The authors declare that there is no conflict of interest.

\section{FINANCIAL SUPPORT}

This work was funded by the National Counsel of Technological and Scientific Development [Conselho Nacional de Desenvolvimento Científico e Tecnológico (CNPq)] scholarship number 142839/2005-1 and Laboratório de Investigação Médica-49, Hospital das Clínicas, Faculdade de Medicina, Universidade de São Paulo (LIM-HC-FMUSP-49).

\section{REFERENCES}

1. Alvar J, Vélez ID, Bern C, Herrero M, Desjeux P, Cano J, et al. Leishmaniasis worldwide and global estimates of its incidence. PLoS One 2012; 7: e35671.

2. Desjeux P. Leishmaniasis: current situation and new perspectives. Comp Immunol Microbiol Infect Dis 2004; 27: 305-318.

3. McGwire BS, Satoskar AR. Leishmaniasis: clinical syndromes and treatment. QJM 2014; 107:7-14.

4. Frézard F, Demicheli C. New delivery strategies for the old pentavalent antimonial drugs. Expert Opin Drug Deliv 2010; 7:1343-1358.

5. Peine KJ, Gupta G, Brackman DJ, Papenfuss TL, Ainslie KM, Satoskar AR, et al. Liposomal resiquimod for the treatment of Leishmania donovani infection. J Antimicrob Chemother 2014; 69:168-175.

6. Schettini DA, Ribeiro RR, Demicheli C, Rocha OG, Melo MN, Michalick MS, et al. Improved targeting of antimony to the bone marrow of dogs using liposomes of reduced size. Int J Pharm 2006; 315:140-147.

7. Alving CR. Liposomes as drug carriers in leishmaniasis and malaria. Parasitol Today 1986; 2:101-107.

8. Carter KC, Dolan TF, Alexander J, Baillie AJ, McColgan C. Visceral leishmaniasis: drug carrier system characteristics and the ability to clear parasites from the liver, spleen and bone marrow in Leishmania donovani infected BALB/c mice. J Pharm Pharmacol 1989; 41:87-91.

9. New RR, Chance ML. Treatment of experimental cutaneous leishmaniasis by liposome-entrapped Pentostam. Acta Trop 1980; 37:253-256.

10. Frézard F, Demicheli C, Ribeiro RR. Pentavalent antimonials: new perspectives for old drugs. Molecules 2009; 14:2317-2336.

11. Schwendener RA, Lagocki PA, Rahman YE. The effects of charge and size on the interaction of unilamellar liposomes with macrophages. Biochim Biophys Acta 1984; 772:93-101.

12. Borborema SET, Schwendener RA, Osso Jr JA, de Andrade Jr HF, do Nascimento N. Uptake and antileishmanial activity of meglumine antimoniate-containing liposomes in Leishmania (Leishmania) major-infected macrophages. Int J Antimicrob Agents 2011; 38:341-347.

13. Tempone AG, Perez D, Rath S, Vilarinho AL, Mortara RA, de Andrade Jr HF. Targeting Leishmania (L.) chagasi amastigotes through macrophage scavenger receptors: the use of drugs entrapped in liposomes containing phosphatidylserine. J Antimicrob Chemother 2004; 54:60-68.

14. Tempone AG, da Silva ACMP, Brandt CA, Martinez FS, Borborema SET, da Silveira MAB, et al. Synthesis and antileishmanial activities of novel 3-substituted quinolines. Antimicrob Agents Chemother 2005; 49:1076-1080.

15. Stewart JC. Colorimetric determination of phospholipids with ammonium ferrothiocyanate. Anal Biochem 1980; 104:10-14.

16. Tada H, Shiho O, Kuroshima K, Koyama M, Tsukamoto K. An improved colorimetric assay for interleukin 2. J Immunol Methods 1986; 93:157-165.

17. Roberts WL, Berman JD, Rainey PM. In vitro antileishmanial properties of tri-and pentavalent antimonial preparations. Antimicrob Agents Chemother 1995; 39:1234-1239.

18. Dey T, Anam K, Afrin F, Ali N. Antileishmanial activities of stearylamine-bearing liposomes. Antimicrob Agents Chemother 2000; 44:1739-1742.

19. Banerjee A, Roychoudhury J, Ali N. Stearylamine-bearing cationic liposomes kill Leishmania parasites through surface exposed negatively charged phosphatidylserine. J Antimicrob Chemother 2008; 61:103-110.

20. Papagiannaros A, Bories C, Demetzos C, Loiseau PM. Antileishmanial and trypanocidal activities of new miltefosine liposomal formulations. Biomed Pharmacother 2005; 59:545-550.

21. Jaafari MR, Bavarsad N, Bazzaz BSF, Samiei A, Soroush D, Ghorbani S, et al. Effect of topical liposomes containing paromomycin sulfate in the course of Leishmania major infection in susceptible BALB/c mice. Antimicrob Agents Chemother 2009; 53:2259-2265.

22. Alving CR, Steck EA, Chapman Jr WL, Waits VB, Hendricks LD, Swartz Jr GM, et al. Therapy of leishmaniasis: superior efficacies of liposome-encapsulated drugs. Proc Natl Acad Sci USA 1978; 75:2959-2963.

23. Rothkopf C, Fahr A, Fricker G, Scherphof GL, Kamps JA. Uptake of phosphatidylserine-containing liposomes by liver sinusoidal endothelial cells in the serum-free perfused rat liver. Biochim Biophys Acta 2005; 1668:10-16.

24. Gilbreath MJ, Hoover DL, Alving CR, Swartz GM Jr, Meltzer MS. Inhibition of lymphokine-induced macrophage microbicidal activity against Leishmania major by liposomes: characterization of the physicochemical requirements for liposome inhibition. J Immunol 1986; 137: 681-1687.

25. Alving CR. Delivery of liposome-encapsulated drugs to macrophages. Pharmacol Ther 1983; 22:407-424.

26. Jousma H, Talsma H, Spies F, Joosten JGH, Junginger HE, Crommelin DJA. Characterization of liposomes. The influence of extrusion of multilamellar vesicles through polycarbonate membranes on particle size, particle size distribution and number of bilayers. Intern J Pharmaceutics 1987; 35:263-274.

27. Olson F, Hunt CA, Szoka FC, Vail WJ, Papahadjopoulos D. Preparation of liposomes of defined size distribution by extrusion through polycarbonate membranes. Biochim Biophys Acta 1979; 557:9-23.

28. Mayer LD, Bally MB, Hope MJ, Cullis PR. Techniques for encapsulating bioactive agents into liposomes. Chem Phys Lipids 1986; 40:333-345.

29. Castro RA, Silva-Barcellos NM, Licio CS, Souza JB, SouzaTestasicca MC, Ferreira FM, et al. Association of liposomeencapsulated trivalent antimonial with ascorbic acid: an effective and safe strategy in the treatment of experimental visceral leishmaniasis. PLoS One 2014; 9:e104055. 
30. Goldbach P, Brochart H, Wehrlé P, Stamm A. Sterile filtration of liposomes: Retention of encapsulated carboxyfluorescein. Int J Pharm 1995; 117:225-230.

31. Guo LS, Hamilton RL, Goerke J, Weinstein JN, Havel RJ. Interaction of unilamellar liposomes with serum lipoproteins and apolipoproteins. J Lipid Res 1980; 21: 993-1003.

32. Cullis PR, Chonn A, Semple SC. Interactions of liposomes and lipid-based carrier systems with blood proteins: Relation to clearance behaviour in vivo. Adv Drug Deliv Rev 1998; 32:3-17.
33. Balasegaram M, Ritmeijer K, Lima MA, Burza S, Ortiz Genovese G, Milani B, et al. Liposomal amphotericin B as a treatment for human leishmaniasis. Expert Opin Emerg Drugs 2012; 17: 493-510.

34. Sundar S, Chakravarty J. Liposomal Amphotericin B and Leishmaniasis: Dose and Response. J Glob Infect Dis 2010; 2:159166. 